\title{
A Model of Handing Interaction Towards a Pedestrian
}

\author{
Chao Shi ${ }^{1,2}$ Masahiro Shiomi ${ }^{1}$ Christian Smith $^{3}$ Takayuki Kanda $^{1}$ Hiroshi Ishiguro ${ }^{1,2}$ \\ ${ }^{1}$ ATR IRC Laboratories \\ 2-2-2 Hikaridai Keihanna Science City \\ Kyoto 610-0228 Japan
1-3 Machikaneyama Toyonaka
Osaka 565-8531 Japan \\ ${ }^{2}$ Osaka University \\ ${ }^{3}$ Royal Institute of Technology, \\ Stockholm, Sweden
}

shi.chao@irl.sys.es.osaka-u.ac.jp, \{m-shiomi, kanda, ishiguro\}@atr.jp, ccs@kth.se

\begin{abstract}
This video reports our research on developing a model for a robot handing flyers to pedestrians. The difficulty is that potential receivers are pedestrians who are not necessarily cooperative; thus, the robot needs to appropriately plan its motion making it is easy and non-obstructive for potential receivers to receive the flyers. In order to establish a model, we analyzed human interaction, and found that (1) a giver approaches a pedestrian from frontal right/left but not frontal center, and (2) he simultaneously stops his walking motion and arm-extending motion at the moment when he hands out the flyer. Using these findings, we established a model for a robot to perform natural proactive handing. The proposed model is implemented in a humanoid robot and is confirmed as effective in a field experiment.
\end{abstract}

Index Terms- Human-robot interaction; handing interaction; interaction during walking

\section{INTRODUCTION}

It is common to see the situation of a shop assistant providing advertisement flyers to visitors, or a clerk distributing pamphlets to the visitors in a museum. Under these circumstances, the shop assistant (or the clerk) who is considered a giver takes the initiative, while the customer (or the visitor) who is considered as a potential receiver initially is not aware of the presence of the giver. Here, it is the potential receiver who chooses whether to act as a receiver to receive the flyer or ignore it and pass by. We expect that a successful giver would make it easy for the potential receivers to receive a flyer while not obstructing their walking, so that potential receivers would feel it easy and comfortable to receive the flyer.

We consider that people non-verbally communicate the intention of handing to potential receivers. Such interaction might be perceived to be easy for humans, however, for a robot it is difficult to replicate what humans unconsciously perform. The robot needs to know every detail of the behavior, such as where and when it should stop and how to make the potential receiver aware of its intention. Hence, we studied handing in human interaction towards a pedestrian, implemented it in a humanoid robot, and confirmed its effectiveness in a field experiment (Figure 1,2).

\section{ACKNOWLEDGMENT}

This research was sponsored by JST, CREST. We thank the administrative staff at the Asia and Pacific Trade Center for their corporation. We also appreciate the assistance of Drazen Brscic, Kotaro Hayashi, Hiroyuki Kidokoro Yoshifumi Nakagawa, and Anderson Li.
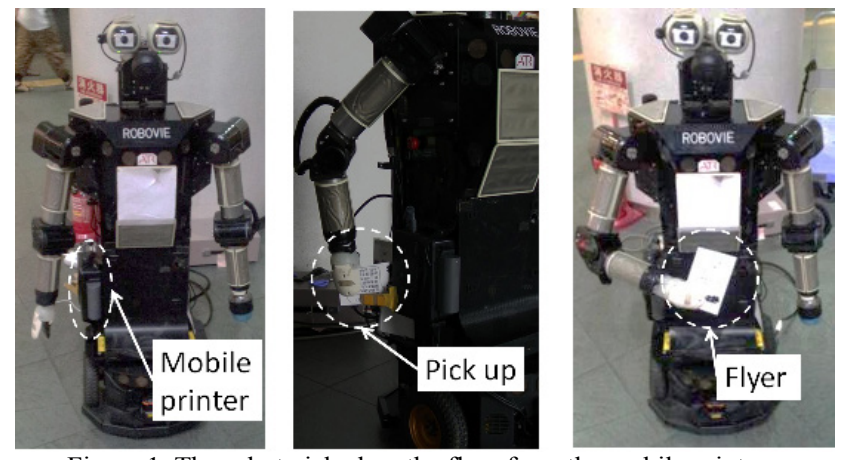

Figure 1. The robot picked up the flyer from the mobile printer
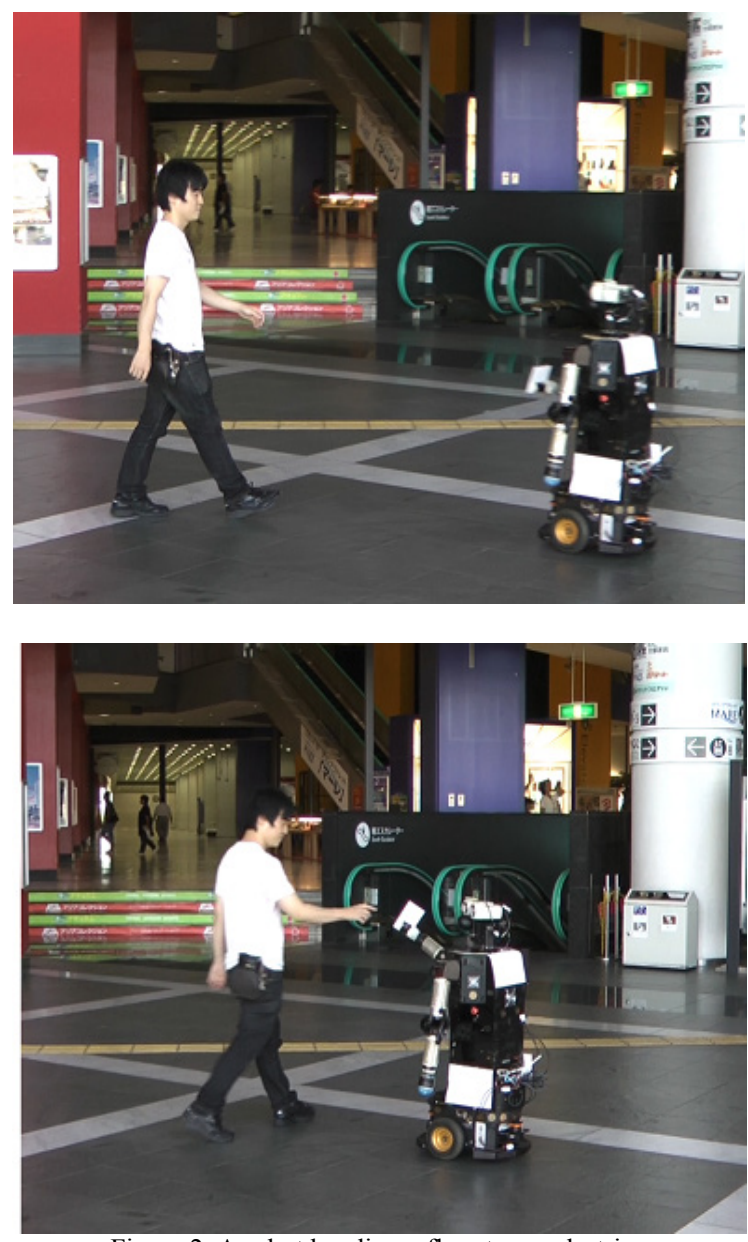

Figure 2. A robot handing a flyer to a pedestrian 